\title{
R-process Nucleosynthesis in Baryon-Rich Jets from Collapsars and Magnetars
}

\author{
Shin-ichiro Fujimoto* \\ Kumamoto National College of Technology, Kumamoto 861-1102, Japan \\ E-mail: fuji@kumamoto-nct.ac.jp
}

Masaomi Ono, and Masa-aki Hashimoto,

Department of Physics, School of Sciences, Kyushu University, Fukuoka 810-8560, Japan

\begin{abstract}
We study $r$-process nucleosynthesis inside baryon-rich jets from collapsars and magnatars, based on two-dimensional magneto-hydrodynamic simulations from the core collapse of stars of $25 \mathrm{M} \odot$ and $40 M_{\odot}$, for magnetar and collapsar models, respectively, to the launch and ejection of jets. We find that the ejecta through the jets become mildly and highly neutron-rich, or electron fraction $Y_{e}>0.3$ and $Y_{e}<0.2$, for the magnetar and collapsar models, respectively. We evaluate abudances of the ejecta for one collapsar model, in which highly neutron-rich material of $Y_{e}<0.2$ is abundantly ejected. We find that heavy $r$-elements are abundantly produced inside the jets and that the abundance profile of the $r$-elements are similar to that of the solar $r$-elements, only for mass number $>120$.
\end{abstract}

XIII Nuclei in the Cosmos,

7-11 July, 2014

Debrecen, Hungary

\footnotetext{
${ }^{*}$ Speaker.
} 


\section{Introduction}

There is still no compelling $r$-process site, although various astrophysical objects have been proposed as the site. In our previous study $[1,2]$, we have proposed a collapsar that is a rotating, massive star, whose central core collapses to a black hole, as a production site of $r$-elements. We have shown that $r$-process successfully operates inside baryon-rich jets from collapsars, in particular jets with high energies [1,2], based on two-dimensional (2D) magneto-hydrodynamic (MHD) simulations from the core collapse of a collapsar to the launch and the ejection of the jets. We also find that low electron fraction, $Y_{e}$, is a key factor for an abundant synthesis of $r$ elements above the second and third-peaks. Nevertheless, we employed a MHD code, in which the $Y_{e}$ evolution is not taken into account. Employed with the same MHD code, we have also shown that $r$-elements above the third-peaks are abundantly synthesized inside baryon-rich jets from a magnetar, that is, a rotating and magnetized, massive star, whose core collapses to a neutron star [3]. Recently, successful production of $r$-elements is obtained for jets launched from a magnetar, based on 3D MHD simulations, employed with a MHD code, in which the $Y_{e}$ evolution is appropriately taken into account [4]. In both studies, magnetars have been set to be relatively low main-sequence masses $\left(\sim 10 M_{\odot}\right)$ and huge magnetic fields $\sim 10^{14} \mathrm{G}$ before the core collapse.

In the present study, we examine $r$-process nucleosynthesis inside the jets ejected from collapsars and magnetars, with a MHD code, in which the $Y_{e}$ evolution is appropriately taken into account. We consider a rotating, magnetized star of $25 M_{\odot}$ with much lower magnetic fields compared with the previous works $[3,4]$ for magnetars, and that of $40 M_{\odot}$ for collapsars.

\section{MHD Simulations of Jets from Collapsars and Magnetars}

We have carried out Newtonian MHD calculations of the collapse of rotating massive stars of $25 M_{\odot}$ and $40 M_{\odot}$ for models of a magnetar and a collapsar, respectively. For magnetar models, MHD calculations are performed in region from the center of a star to an outer boundary. On the other hand, for collapsar models, the core of the star is assumed to be collapsed to a black hole promptly. Calculations are performed over the region other than the central region $(\leq 50 \mathrm{~km})$ of the star. Fluid is freely absorbed through the inner boundary of $20 \mathrm{~km}$, which mimics a surface of the black hole. The outer boundary of the MHD calculation is set to be $10,000 \mathrm{~km}$ from the center of the stars. The numerical code for the MHD calculations is based on the ZEUS-2D code, as in our previous works $[1,2]$, in which the $Y_{e}$ evolution is not followed but is determined with postprocessing manner for ejecta. In the current study, however, we follow the evolution in the MHD simulations, taking into account the change in $Y_{e}$ due to electron and positron capture and neutrino and anti-neutrino absorption in terms of a leakage scheme $[5,6]$.

Spherical coordinates, $(r, \theta, \phi)$ are used in our simulations and the computational domain is extended over $20 \mathrm{~km} \leq r \leq 10000 \mathrm{~km}$ and $0 \leq \theta \leq \pi / 2$ and covered with $250(r) \times 24(\theta)$ meshes for collapsars. For magnetars, the computational domain is extended over $0 \mathrm{~km} \leq r \leq 10000 \mathrm{~km}$ and $0 \leq \theta \leq \pi / 2$ and covered with $300(r) \times 48(\theta)$ meshes. We assume the fluid is axisymmetric and the mirror symmetry on the equatorial plane. We mimic strong gravity around the black hole in terms of the pseudo-Newtonian potential. 
We set the initial profiles of the density, temperature and $Y_{e}$ to those of the spherical model of $25 M_{\odot}$ and $40 M_{\odot}$ massive stars before the collapse [7], We adopt an analytic form of the angular velocity $\Omega(r)$ of the star before the collapse: $\Omega(r)=\Omega_{0} \frac{R_{0}^{2}}{r^{2}+R_{0}^{2}}$, as in our previous work. Here $\Omega_{0}$ and $R_{0}$ are parameters of our model. We consider three sets of $\left(\Omega_{0}, R_{0}\right)=(5 \mathrm{rad} / \mathrm{s}, 1400 \mathrm{~km})$ (a model with rapidly rotating core, or a $\mathrm{R}$ model), $(2.5 \mathrm{rad} / \mathrm{s}, 2000 \mathrm{~km})$ (a model with moderately rotating core, or a M model), and, (1.0 rad $/ \mathrm{s}, 3200 \mathrm{~km}$ ) (a model with slowly rotating core, or a $\mathrm{S}$ model). We note that in our previous work [1,2], we have referred to models with $\left(\Omega_{0}, R_{0}\right)=(10 \mathrm{rad} / \mathrm{s}$, $1000 \mathrm{~km})$ as R models and those with $\left(\Omega_{0}, R_{0}\right)=(0.5 \mathrm{rad} / \mathrm{s}, 5000 \mathrm{~km})$ as $\mathrm{S}$ models. For the sets of $\Omega_{0}$ and $R_{0}$, the maximum specific angular momentum is around $10^{17} \mathrm{~cm}^{2} / \mathrm{s}$, which is comparable to that of the Keplerian motion at $50 \mathrm{~km}$ around a black hole of $3 M_{\odot}$. Therefore the centrifugal force can be larger than the gravitational force of the central black hole and the formation of disk like structure is expected near the hole for the collapsar models.

Initial magnetic field is assumed to be uniform, parallel to the rotational axis, and weak elsewhere $\left(\beta=8 \pi P / B^{2} \gg 1\right)$. In the present paper, we set initial magnetic fields, $B_{0}=10^{10}$ and $10^{12} \mathrm{G}$ for the collapsar and magnetar models, respectively. It should be noted that the magnetic pressure is much smaller than the other pressure before the collapse even if $B_{0}$ is equal to $10^{12} \mathrm{G}$. In brief, we consider three collapsar- and two magnetar-models in the present study, that is cR10, cM10, and cS10 (collapsar models), mR12 and mM12 (magnetar model), in which the first-lower and second-upper characters are refereed as a progenitor type (c(=collapsar) or m(=magnetar)) and rotation (R, M, or S), respectively, and the last number (10 or 12) equals to $\log B_{0}$.

We briefly describe results of our MHD calculation. All five models reveals the formation of jets due to magnetic fields, which are dominated by the toroidal component at an epoch of the jet formation and are chiefly amplified due to the wrapping of the field inside an accretion disk for the collapsar models or a neutron star for the magnetar models. For the collapsar models, the disk become neutron-rich due to electron captures on protons, which produce neutrons and electron-type neutrinos (left panel of Fig. 1) and such the neutron-rich material inside the disk is ejected through the jet (right panel of Fig. 1). In particular for cM10 and cS10 models, the jets become highly neutron-rich enough to synthesize a large amount of $r$-process elements around the third-peak, as shown in later.

\section{Results}

\subsection{Neutron-richness of ejecta}

In order to calculate the chemical composition of ejecta through jets, we need Lagrangian evolution of physical quantities, such as density, temperature, $Y_{e}$ and, velocity of the ejecta. We adopt a tracer-particle method, as in $[1,2]$ to calculate the Lagrangian evolution from the Eulerian evolution obtained from our MHD calculations. Particles are initially placed from the Fe core to an inner O-rich layer. The total numbers of the particles are set to be $N_{p}=10,000$, with which we can follow ejecta through the jets appropriately. We have already examined how different $N_{p}$ change masses and abundances of ejecta through the jets in our previous work [1].

High neutron-richness, or low $Y_{e}<0.2-0.3$, is a key ingredient in the ejecta for successful $r$-process, through which a significant amount of third-peak elements is synthesized, since the jets 
Time: $0.1980 \mathrm{sec}$

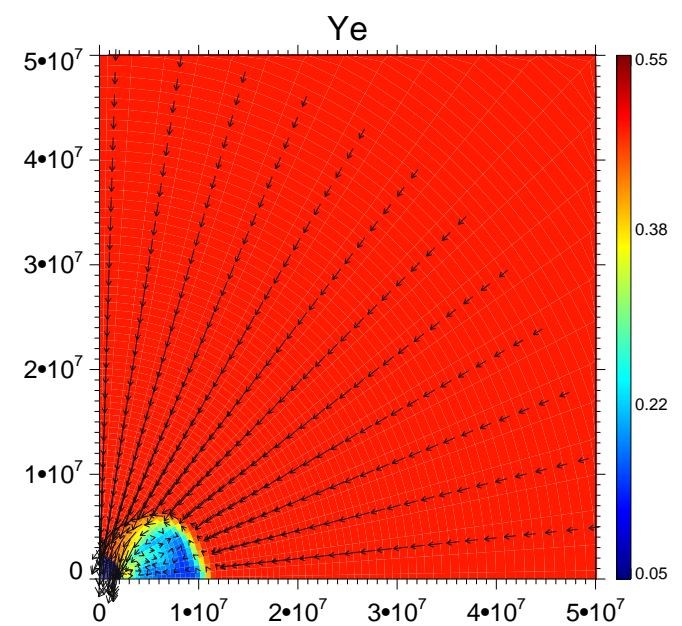

Time: $0.4828 \mathrm{sec}$

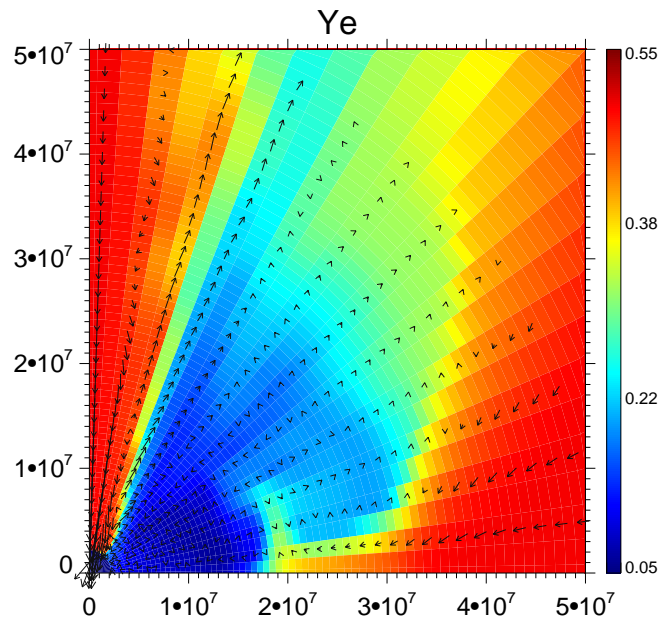

Figure 1: $Y_{e}$ contour for $\mathrm{cM} 10$ model at $0.20 \mathrm{~s}$ (left panel) and $0.48 \mathrm{~s}$ (right panel) after the core collapse of the progenitor. The accretion disk is formed around the black hole and neutron-rich (or low $Y_{e}$ ) (left panel). The jet is enough neutron-rich (right panel) for third-peak elements to be produced through strong $r$-process.

are baryon-rich and have a moderate value of entropy per baryon $<20$ in units of the Boltzmann constant $k_{\mathrm{B}}$, contrast to a condition for successful $r$-process in neutrino-driven winds from a neutron star; the ejecta is required to be $Y_{e}$ around 0.4 with very high entropy $>(200-300) k_{\mathrm{B}}$ for the winds.
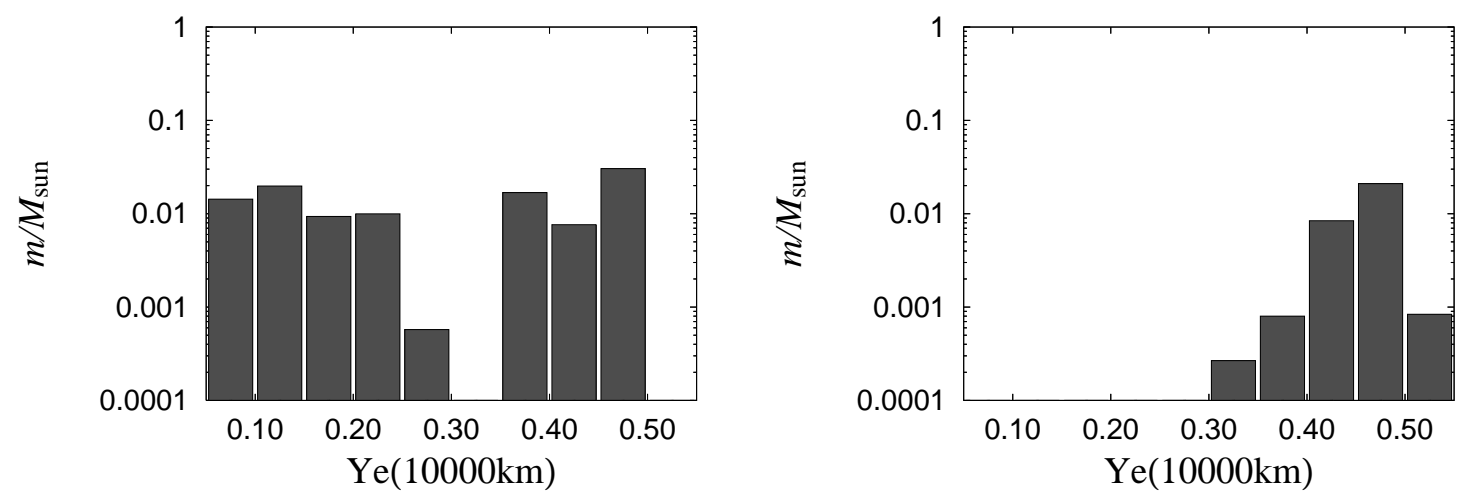

Figure 2: $Y_{e}$ of the ejecta as a function of masses in units of $M_{\odot}$ for a collapsar model, cM10 (left panel) and a magnetar model, mM12 (right panel). $Y_{e}$ is evaluated at a position where a tracer particle reaches $10,000 \mathrm{~km}$ from the center.

We find that $Y_{e}$ of the ejecta through the neutron-rich disk and the jets (Fig. 1) becomes less than 0.2 for collapsar models. Figure 2 shows $Y_{e}$ distribution of the ejecta for cM10 (left panel) and for mM12 (right panel), as a function of masses. Low $Y_{e}<0.2$ material is found to be abundantly ejected through the jets for the collapsar model, cM10. We note that the model, cM10 is the most abundant model of neutron-rich material $\left(Y_{e}<0.3\right)$ with mass of $\sim 0.05 M_{\odot}$, compared with the other four models. For $\mathrm{cS} 10$, low $Y_{e}<0.2$ is found to be ejected but has a much lower mass 
compared with that for $\mathrm{cM} 10$. The ejecta is however mildly neutron-rich, or $Y_{e}>0.3$ for $\mathrm{cR} 10$.

For two magnetar models, the ejecta becomes mildly neutron-rich with $Y_{e}>0.3$ (see right panel of Fig. 2), since only material near the surface of the neutron star $Y_{e}>0.3$ is ejected through the jets. Contrary to the present study, low $Y_{e}$ material is ejected in the previous works [3, 4], in which highly neutron-rich matter of $Y_{e}<0.2$ is ejected from the central part of stars, whose mass is relatively low $\sim(10-13) M_{\odot}$ with huge magnetic fields $\left(\sim 10^{14} \mathrm{G}\right)$ before the core collapse.

\subsection{Abundances of ejecta}

We follow the evolution of abundances of an ejected tracer particle through the jets using a large nuclear-reaction network [1] with the Lagrangian evolution of physical quantities of the ejected particle, whose evolution of the physical quantities have been followed in terms of the tracer particle method. We sum the mass fractions of ejected particles weighted by their mass over all the ejected tracer particles to get the integrated abundances of the ejecta. In the present study, we have calculated the abundances of the ejecta only for $\mathrm{cM} 10$, since the ejecta have a large fraction of highly neutron-rich material, whose mass is significant $\sim 0.05 M_{\odot}$.
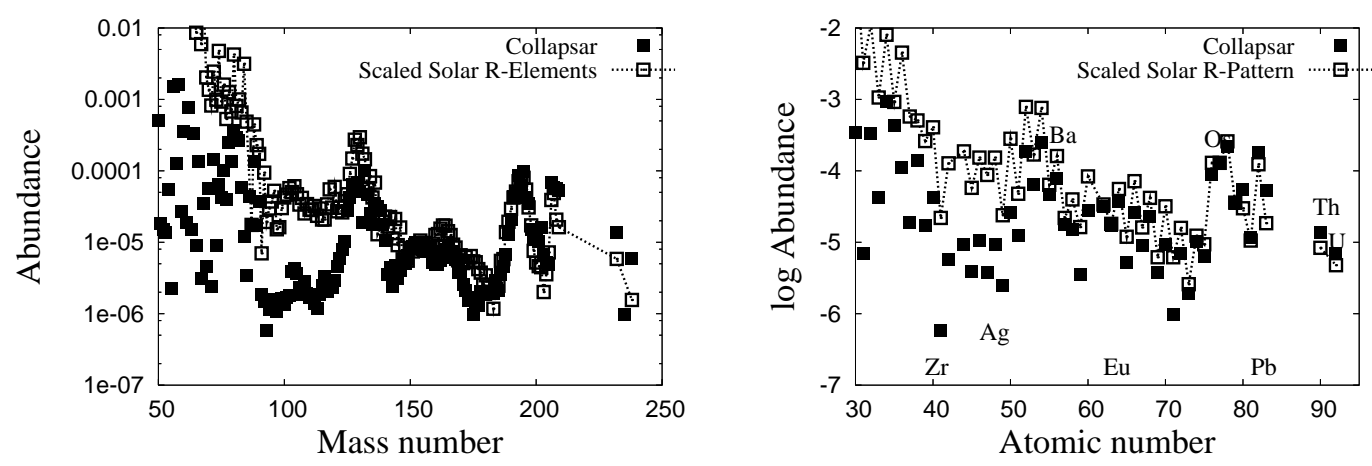

Figure 3: Integrated abundances of the ejecta as a function of mass numbers (left panel) and atomic numbers (right panel) for cM10 model. Abundances of $r$-process elements heavier than the second peak and atomic number $>50$ are similar to those of the solar system. $R$-process elements lighter than the second peak, however, are underproduced compared with the solar-relements.

Figure 3 shows integrated abundances of the ejecta (filled-squares) as a function of mass numbers (left panel) and atomic numbers (right panel). Abundances of $r$-process elements heavier than the second peak and atomic number $>50$ are similar to the profile of the solar-relements, which are described with open-squares in Fig. 3. $R$-process elements lighter than the second peak, however, are underproduced compared with the solar-relements, due to the smallness of the fraction of mildly neutron-rich ejecta $\left(Y_{e}>0.3-0.47\right)$, as shown in Fig. 2.

For cS10, a fraction of mildly neutron-rich ejecta $\left(Y_{e}>0.3-0.47\right)$ is higher than that for cM10, abundances could be much similar to profiles of the solar $r$-elements with a much smaller mass of ejected $r$-elements. For the magnetar models, no third-peak elements is possibly produced due to no highly neutron-rich ejecta with $Y_{e}<0.3$.

\subsection{Neutrino capture on nucleons in ejecta}

We briefly discuss effects of neutrino capture on neutrons and protons in ejecta on the pro- 
duction of $r$-elements. A fraction of neutrinos emitted from disks or neutron stars is absorbed by nucleons in the ejecta through jets. The neutrino absorption might decrease their neutron-richness or to increase $Y_{e}$ to be inefficient for $r$-process. The neutrino luminosities are evaluated from our MHD simulations and found to be $\sim 1$ and $\sim 4 \times 10^{52} \mathrm{erg} \mathrm{s}^{-1}$ for the collapsar and magnetar models, respectively. For the collapsar models, the neutrinos are emitted from the accretion disk, which is located near the equatorial plane around the black hole, which does not emit neutrinos. The neutrino luminosities are therefore smaller than those for the magnetar models, in which neutrinos are efficiently emitted from the central neutron star. For cM10, the ejecta could remain low $Y_{e}<0.2$ for such relatively low neutrino luminosity $\sim 1 \times 10^{52} \mathrm{erg} \mathrm{s}^{-1}$, even if neutrino absorption on nucleons would be taken into account. We note that the luminosity is comparable to those for neutrino-driven winds from the surface of a neutron star but the jets are much faster than the winds. The neutrino absorption in the jets is possibly less important compared with the winds.

\section{Summary}

We have examined $r$-process nucleosynthesis inside jets from magnetars and collapsars, based on 2D MHD simulations, from the core collapse of stars of $25 M_{\odot}$ and $40 M_{\odot}$, for two magnetar and three collapsar models, respectively, to the launch and ejection of jets, for a given set of distributions of angular velocities and magnetic fields. We find that the ejecta through the jets become mildly and highly neutron-rich, or $Y_{e}>0.3$ and $Y_{e}<0.2$, for the magnetar and collapsar models, respectively, and for the collapsar models, such low $Y_{e}$ could remain, even if the neutrino absorption would be taken into account, because of the low neutrino luminosities. We followed the abundance evolution of the ejecta for one collapsar model, in which highly neutron-rich material of $Y_{e}<0.2$ is abundantly ejected, and find that heavy $r$-elements are abundantly produced inside the jets and that the profile of the $r$-elements are similar to that of the solar $r$-elements, only for mass number $>120$.

\section{Acknowledgments}

This work was supported in part by a Grant-in-Aid for Scientific Research from the Ministry of Education, Culture, Sports, Science and Technology of Japan (No. 22540297).

\section{References}

[1] Fujimoto, S., Hashimoto, M., S., Kotake, K., \& Yamada, S., 2007, ApJ 656, 382

[2] Fujimoto, S., Nishimura, N., \& Hashimoto, M., 2008, ApJ 680, 1350

[3] Nishimura, S., Kotake, K., Hashimoto, M.-a., et al., 2006, ApJ 642, 410

[4] Winteler, C., Kappeli, R., Perego, A., et al.,2012, ApJ L750, L22

[5] Ruffert, M., Janka, H.-T., \& Schaefer, G. 1996, A\&A, 311, 532

[6] Kotake, K., Sawai, H., Yamada, S., \& Sato, K. 2004, ApJ , 608, 391

[7] Hashimoto, M., 1995, Progress of Theoretical Physics, 94, 663 